\title{
Wellness centres: an important but overlooked source of Legionnaires' disease. Eight years of source investigation in the Netherlands, 1 August 2002 to 1 August 2010
}

S M Euser (s.euser@streeklabhaarlem.nl) ${ }^{1}$, J P Bruin ${ }^{1}$, W van der Hoek ${ }^{2}$, W A Schop ${ }^{3}$, J W den Boer ${ }^{1}$

1. Regional Public Health Laboratory Kennemerland, Haarlem, the Netherlands

2. National Institute for Public Health and the Environment, Bilthoven, The Netherlands

3. Municipal Public Health Service Rotterdam-Rijnmond, Rotterdam, the Netherlands

Euser SM, Bruin JP, van der Hoek W, Schop WA, den Boer JW. Wellness centres: an important but overlooked source of Legionnaires' disease. Eight years of source investigation in the Netherlands, 1 August 2002 to 1 August 2010.

Euro Surveill. 2012;17(8):pii=20097. Available online: http://www.eurosurveillance.org/ViewArticle.aspx?Articleld=20097

Article published on 23 February 2012

Visiting wellness centres is considered safe and relaxing and might provide health benefits for visitors with certain cardiovascular, dermatological or respiratory diseases. On the other hand, wellness centres could pose health risks, especially with respect to Legionnaires' disease. We investigated the role of wellness centres in the occurrence of Legionnaires' disease by analysing the data of eight years (20022010) of source investigation in the Netherlands. There were 15 wellness centres identified as potential sources of infection for a total of 35 Legionnaires' disease patients. Twelve of these centres were positive for Legionella spp.: six for Legionella pneumophila, six for non-pneumophila Legionella spp.. Of the 65 positive environmental samples found during the wellness centre investigations, 41 were derived from shower heads. For two centres, the Legionella pneumophila strains in the collected samples had a genotype that was indistinguishable from the patient isolates. These results show that wellness centres are potential sources of Legionnaires' disease.

\section{Introduction}

Apart from massages and beauty care most wellness centres offer a mix of saunas, swimming pools, whirlpools, and other bathing facilities to the general public. Visiting these wellness centres is considered safe and relaxing and might even provide health benefits for visitors with certain cardiovascular, dermatological or respiratory diseases $[1,2]$. On the other hand, is has been shown that facilities with whirlpools or saunas could comprise health risks, for example with respect to Legionnaires' disease [3-5]. This acute pneumonia is caused by Legionella spp., which are thought to be responsible for two to $15 \%$ of all communityacquired pneumonias [6-8]. Legionella spp. live in aquatic environments and are particularly prevalent in man-made habitats [9]. The major route of transmission for Legionnaires' disease is inhalation of the bacterium that is spread into the air as an aerosol from either natural or man-made sources [10]. Modern use of devices that aerosolise water or settings with such devices (e.g. air conditioners, showers, cooling towers, fountains, wellness centres), largely contribute to the emergence of Legionnaires' disease as an important waterborne disease.

Previous reports showed that in several cases of Legionnaires' disease, wellness centres (with saunas and/or whirlpools) were indeed identified as the source of infection [3-5]. However, further clarification of the role of these centres in Legionella infections warrants a systematic identification and investigation of potential sources of Legionnaires' disease. In 2002, based on the observation that outbreaks of Legionnaires' disease are often preceded and followed by small clusters of cases [11], the Netherlands established the Legionella Source Identification Unit (LSIU) as part of a National Legionella Outbreak Detection Programme (NLODP) [12]. The aim of this programme was to improve source identification, thereby preventing or controlling outbreaks of Legionnaires' disease by swift elimination of the source.

In this study we aimed to assess the importance of wellness centres in the occurrence of Legionnaires' disease by analysing the data of eight years (2002-2010) of systematic source investigation within the NLODP in the Netherlands.

\section{Methods \\ National Legionella Outbreak \\ Detection Programme}

As part of the NLODP, a LSIU was available to all Municipal Health Services for sampling of potential sources of Legionella infection in reported cases of Legionnaires' disease. Between 2002 and 2006, all identified potential sources of infection were 
investigated. From 2006 onwards, the LSIU has only investigated potential sources if at least one of the following four sampling-criteria was met: (i) A patient isolate of Legionella spp. from respiratory secretions or lung tissue is available; (ii) one of the potential sources of infection identified by a Legionnaires' disease patient was previously identified as a potential source of a different Legionnaires' disease patient; (iii) the residence of a reported Legionnaires' disease patient is situated within a range of less than one kilometre from the residences of at least two other Legionnaires' disease patients who were reported in the last six months; (iv) the patient stayed in a hospital during the incubation period.

\section{Patients}

Legionnaires' disease has been notifiable in the Netherlands since 1987 . Treating physicians are required to report cases of Legionnaires' disease to a public health physician at one of the 29 Municipal Health Services within 24 hours of diagnosis. The public health physicians are then required to report all confirmed and probable cases of Legionnaires' disease to the Ministry of Health and, since 2006, to the Centre for Infectious Disease Control, within 24 hours. A confirmed case of Legionnaires' disease is defined as a patient suffering from symptoms compatible with pneumonia, with radiological signs of infiltration, and with laboratory evidence of Legionella spp. infection (including isolation of Legionella spp. from respiratory secretions or lung tissue, detection of L. pneumophila antigen in urine, seroconversion or a four-fold or higher rise in antibody titres to Legionella spp. in paired acute- and convalescent-phase sera). A probable case of Legionnaires' disease is defined as a patient suffering from symptoms compatible with pneumonia, with radiological signs of infiltration, and with laboratory findings suggestive of Legionella spp. infections (including a high antibody titre to Legionella spp. in a single serum, direct fluorescent antibody staining of the organism or detection of Legionella species DNA by polymerase chain reaction (PCR) in respiratory secretions or lung tissue). All 62 microbiological laboratories in the Netherlands involved in the diagnosis and treatment of patients with pneumonia are requested to send the available isolates of Legionella spp. from respiratory secretions or lung tissue of patients to the LSIU.

Given the purpose of the programme to identify Dutch sources of infection, patients who had stayed abroad for five days or more during their incubation period of two to 10 days were not considered for source identification.

\section{Source identification and sampling procedure}

Potential sources of infection were identified by public health physicians and nurses from the Municipal Health Service who interviewed the patient and/or a relative. The interview focused on tracking each patient's exposure to potential sources of infection during the two weeks before their first symptoms occurred. If at least one of the four sampling criteria was met, trained laboratory staff from the LSIU took water and swab samples from the identified potential sources. For each location, sampling points were selected by the LSIU staff in cooperation with the technical team of a facility (when available) to obtain a comprehensive collection of water and swab samples for further analysis. The sampling procedure was in accordance with national guidelines $[13,14]$. It is noteworthy that the LSIU sampling method differs slightly from the European guidelines, which recommend samples of one litre in volume to be collected immediately after the opening of the water outlet [15], while the LSIU samples $500 \mathrm{ml}$ in volume.

\section{Laboratory investigations}

The water samples were concentrated by filtration and filtered residues were resuspended in $1 \mathrm{ml}$ sterile water. Of this suspension, $100 \mu \mathrm{l}$ samples were cultured without dilution and after 10 -fold dilution on two media at $35^{\circ} \mathrm{C}$, with increased humidity. The two media used were buffered charcoal yeast extract supplemented with a-ketogluterate (BCYE-a) and (i) the antibiotics polymyxin $B$, cefazolin, and pimaricin; and (ii) the antibiotics polymyxin B, anisomysin, and vanomycin. In cases of bacterial overgrowth, cultures were repeated after pre-treatment by heating 30 minutes at $50^{\circ} \mathrm{C}$. Swab samples were dispersed by immersion in $1 \mathrm{ml}$ sterile water and cultured as described above. Both patient and environmental Legionella isolates were serogrouped by using commercially available kits containing antisera against L. pneumophila serogroups $1-14$, L. longbeachae 1 and 2, L. bozemanii 1 and 2, L. dumoffii, L. gormanii, L. jordanis, L. micdadei, and L. anisa (Legionella latex test, Oxoid Limited, Hampshire, England; Legionella antisera "Seiken," Denka Seiken Co. Ltd., Tokyo, Japan). All Legionella pneumophila serogroup 1 strains that were found in patient isolates or in the collected samples were subsequently genotyped by amplified fragment length polymorphism (AFLP) analysis, and by sequence based typing (SBT), as recommended by the European Working Group for Legionella Infections (EWGLI) [1618]. Patient isolates were then compared with environmental strains that were found in the samples of potential sources that were investigated.

\section{Control measures}

Whenever a wellness centre was found positive for Legionella spp. after sampling, the responsible government agency (usually the Inspectorate of the Ministry of Housing, Spatial Planning and the Environment (VROM Inspectorate)) was informed by the Municipal Health Services. They assessed how codes of practice and legal regulations concerning the prevention of Legionnaires' disease had been followed, and recommended or enforced control measures such as thermal or chemical disinfection and adaptation of the plumbing system to prevent new cases of Legionnaires' disease. 


\section{Results}

\section{Patients}

From 1 August 2002 until 1 August 2010, 2,076 confirmed or probable cases of Legionnaires' disease were notified to the Centre for Infectious Disease Control. The 619 (30\%) patients who had stayed abroad for five days or more during their incubation period (2-10 days) were excluded from the analyses. The remaining 1,457 patients were investigated by the Municipal Health Services and the LSIU. Patient characteristics are shown in Table 1. Patients had a median age of 59.5 (interquartile range (IQR): $50.7-70.0$ ) years, and $29 \%$ were female.

The 2,343 potential sources of infection that were mentioned by the patients during the interviews with the Municipal Health Service are shown in Table 2. Patient homes were mentioned by the majority of patients, followed by garden centres, workplaces, hospitals, cooling towers, and sports facilities. Wellness centres ranked 11th on the list of most often mentioned potential sources.

\section{Source investigation}

Source investigation resulted in the sampling of 1,317 of the 2,343 potential sources by the LSIU that were related to one or more of the 1,457 patients. Some of the potential sources were more frequently associated with Legionella findings than others, which is reflected in the proportion of investigations where Legionella was found in the investigated source. The sampling results are shown in Table 3, where the sources are ranked by the percentage of positive source investigations (from high to low). It should be noted that an individual source was sometimes investigated more than once (some sources were repeatedly identified by new patients during the study period). The proportion of potential source investigations that were positive for Legionella spp. was highest for wellness centres (28 of 33 source investigations), followed by cooling towers, hospitals, hotels, swimming pools, sports facilities, holiday parks, and home residences (Table 3).

When the different species of Legionella are considered, the data show that in 21 of the 33 wellness centre investigations Legionella pneumophila was found in one or more of the investigated samples, ranking

\section{TABLE 1}

Probable or confirmed cases of Legionnaires' disease, by age group, the Netherlands, 1 August 2002-1 August 2010 $(\mathrm{n}=1,457)$

\begin{tabular}{|l|c|c|}
\hline Age group (years) & Female $\mathrm{n}(\%)$ & Male $\mathrm{n}(\%)$ \\
\hline $0-25$ & $7(1.7)$ & $8(0.8)$ \\
\hline $26-50$ & $87(20.7)$ & $238(23.0)$ \\
\hline $51-75$ & $244(58.1)$ & $640(61.7)$ \\
\hline 75 & $82(19.5)$ & $151(14.6)$ \\
\hline Total & $420(100.0)$ & $\mathbf{1 , 0 3 7 ( 1 0 0 . 0 )}$ \\
\hline
\end{tabular}

wellness centres first before cooling towers, hospitals, hotels, swimming pools, sport facilities, and holiday parks (Table 3). The majority of the 65 positive samples found during the wellness centre investigations were derived from shower heads $(n=41)$. Other positive sample locations within the wellness centres were: taps $(n=12)$ and whirlpools $(n=3)$.

The 33 investigations of wellness centres were performed at 15 unique sites. Twelve of these centres were positive for Legionella spp. (six centres for Legionella pneumophila, and six centres for non-pneumophila Legionella spp.). The number of investigations on individual wellness centres testing positive for Legionella spp. ranged from one to seven. The 15 investigated wellness centres were identified by 35 patients, of whom 25 were part of different clusters associated with seven large and small wellness centres all positive for Legionella. There was one wellness centre with seven clustered patients, two centres with four patients, two centres with three patients, and two centres with two patients.

\section{Genotype comparison}

For 129 of the 333 positive source investigations that were performed between 2002 and 2010, there was a patient isolate available for genotyping which allowed comparison with the genotypes of the environmental strains found in the samples. In 33 cases the available patient isolate had an indistinguishable genotype from those of the environmental strains reflecting a success rate of $25 \%(33 / 129)$. The majority of these 'matches' were made with strains from investigated hospitals (13 matches of 13 positive investigation with an available patient isolate), home residences (nine matches of 47), hotels (two matches of two), swimming pools (two matches of seven), and wellness centres (two matches of 13).

\section{TABLE 2}

Potential sources of infection $(n=2,343)$ reported by Legionnaires' disease cases $(n=1,457)$, the Netherlands, 1 August 2002-1 August 2010

\begin{tabular}{|l|c|}
\hline Reported potential source of infection & $\mathrm{n}(\%)$ \\
\hline Home residence & $1,149(49.0)$ \\
\hline Garden centre & $146(6.2)$ \\
\hline Workplace & $138(5.9)$ \\
\hline Hospital & $115(4.9)$ \\
\hline Cooling tower & $89(3.8)$ \\
\hline Sports facility & $68(2.9)$ \\
\hline Swimming pool & $59(2.5)$ \\
\hline Holiday park & $48(2.0)$ \\
\hline Hotel & $47(2.0)$ \\
\hline Car wash installation & $47(2.0)$ \\
\hline Wellness centre & $44(1.9)$ \\
\hline Campsite & $39(1.7)$ \\
\hline Fountain & $38(1.6)$ \\
\hline Other & $316(13.5)$ \\
\hline
\end{tabular}




\section{Discussion}

Given the low ranking of potential sources mentioned by Legionnaires' disease patients, wellness centres do not seem to contribute much to Legionnaires' disease transmission. However, our data show that in $85 \%$ (28 of 33) of all investigations wellness centres were positive for Legionella spp. This rate is remarkably higher compared to other types of potential sources like cooling towers (18 of $33(55 \%)$ ), hospitals (34 of $68(50 \%)$ ), homes (139 of $693(20 \%)$ ) and garden centres (eight of $63(13 \%))$ that were identified, investigated and sampled under identical conditions. Moreover, typing results indicate that in more than $60 \%$ (six of 33 ) of all wellness centre investigations, Legionella pneumophila, which is thought to be the etiologic agent in over $90 \%$ of all Legionnaires' disease patients [19], was found in at least one of the samples. Compared to the other potential sources that were investigated, wellness centres account for the highest percentage of Legionella pneumophila positive source investigations, which further indicates the relatively high potential of wellness centres as sources of Legionnaires' disease.

There are several possible explanations for our findings. One of them is that the circumstances in wellness centres contribute to a Legionella-friendly environment. The abundant presence of showers, whirlpools, swimming pools and even air-perfused footbaths can clearly form a Legionella-friendly habitat and lead to free Legionella in the air. Additionally, the complexity of water piping systems due to subsequent enlargements of wellness centres could lead to standing or slow-flowing water and thereby create a stable microenvironment for growth of Legionella.

Another possibility is that the visitors of wellness centres may be more at risk for Legionnaires' disease compared to individuals who do not visit these centres. Underlying chronic diseases and smoking status are known risk factors for Legionnaires' disease [20]. If an overrepresentation of individuals who are at higher risk for Legionnaires' disease among wellness centres visitors is confirmed, a possible public health intervention would be to inform this group on the risks of wellness recreation. We were unfortunately not able to study this possibility in the current study setting. However, considering the remarkable source investigation results we do think that there is a role awaiting for public health education aimed at wellness centre visitors who are at increased risk for Legionnaires' disease.

It is difficult to compare our results with previous European studies on surveillance of Legionnaires' disease because of the absence of a systematic source identification and investigation programme in other countries. Although several outbreak reports have acknowledged wellness centres as an important

\section{TABLE 3}

Results of investigations $(n=1,317)$ of potential sources of infection reported by Legionnaires' disease cases $(n=1,457)$, the Netherlands, 1 August 2002-1 August 2010

\begin{tabular}{|c|c|c|c|c|c|c|}
\hline \multirow{3}{*}{ Source type $(n)^{a}$} & \multicolumn{6}{|c|}{ Number of investigations ${ }^{\mathrm{b}}$ for Legionella spp. } \\
\hline & \multicolumn{4}{|c|}{ Positive for Legionella spp. } & \multirow{2}{*}{$\begin{array}{c}\text { Negative for } \\
\text { Legionella spp. } \\
\text { Total negative } \\
\text { n (\%) }\end{array}$} & \multirow{2}{*}{$\begin{array}{l}\text { Total } \\
\text { Total } \\
\text { n (\%) }\end{array}$} \\
\hline & $\begin{array}{c}\text { L. pneumophila } \\
\text { n (\%) }\end{array}$ & $\begin{array}{c}\text { non-pneumophila } \\
\text { Legionella spp. } \\
\text { n (\%) }\end{array}$ & $\begin{array}{l}\text { L. pneumophila and } \\
\text { non-pneumophila } \\
\text { Legionella spp. } \mathrm{n}(\%)\end{array}$ & $\begin{array}{c}\text { Total positive } \\
\text { n (\%) }\end{array}$ & & \\
\hline Wellness centre $(n=15)$ & $15(45)$ & $7(21)$ & $6(18)$ & $28(85)$ & $5(15)$ & $33(100)$ \\
\hline Cooling tower $(n=30)$ & $15(45)$ & $2(6)$ & $1(3)$ & $18(55)$ & $15(45)$ & $33(100)$ \\
\hline Hospital $(n=48)$ & $14(21)$ & $15(22)$ & $5(7)$ & $34(50)$ & $34(50)$ & $68(100)$ \\
\hline Hotel $(n=14)$ & $3(20)$ & $2(13)$ & $1(7)$ & $6(40)$ & $9(60)$ & $15(100)$ \\
\hline Swimming pool $(n=32)$ & $5(15)$ & $5(15)$ & $2(6)$ & $12(35)$ & $22(65)$ & $34(100)$ \\
\hline Sports facility $(n=26)$ & $4(15)$ & $3(12)$ & $1(4)$ & $8(31)$ & $18(69)$ & $26(100)$ \\
\hline Holiday park $(\mathrm{n}=19)$ & $3(14)$ & $3(14)$ & $\mathrm{o}(0)$ & $6(27)$ & $16(73)$ & $22(100)$ \\
\hline Other $(n=199)$ & $19(9)$ & $31(15)$ & $3(1)$ & $53(26)$ & $150(74)$ & $203(100)$ \\
\hline Home residence $(n=693)$ & $39(6)$ & $93(13)$ & $7(1)$ & $139(20)$ & $554(80)$ & $693(100)$ \\
\hline Workplace $(n=78)$ & $6(7)$ & $8(10)$ & $2(2)$ & $16(20)$ & $66(80)$ & $82(100)$ \\
\hline Car wash installation $(n=11)$ & $\mathrm{o}(\mathrm{o})$ & $2(18)$ & $\mathrm{o}(0)$ & $2(18)$ & $9(82)$ & $11(100)$ \\
\hline Garden centre $(n=51)$ & $2(3)$ & $6(10)$ & $\mathrm{o}(\mathrm{o})$ & $8(13)$ & $55(87)$ & $63(100)$ \\
\hline Fountain $(n=11)$ & $\mathrm{o}(0)$ & $1(9)$ & $o(0)$ & $1(9)$ & $10(91)$ & $11(100)$ \\
\hline Campsite $(n=23)$ & $1(4)$ & $1(4)$ & $\mathrm{o}(0)$ & $2(9)$ & $21(91)$ & $23(100)$ \\
\hline Total $(n=1,250)$ & $126(10)$ & 179 (14) & $28(2)$ & $333(25)$ & $984(75)$ & $1,317(100)$ \\
\hline
\end{tabular}

L. pneumophila: Legionella pneumophila.

This number represents the number of unique sources.

b A unique source could be the subject of more than one investigation if it was repeatedly identified by Legionnaires' disease cases over the eight year period covered by this study. 
source of exposure in Legionnaires' disease outbreaks $[4,5]$, most European surveillance programmes do not include these specific potential sources in their surveillance data $[21,22]$. The installation of a European surveillance programme in which systematic environmental investigations are incorporated could elucidate the role of different potential sources in Legionnaires' disease cases.

The strengths of this study are the nationwide detection and registration of new Legionnaires' disease cases and additional source identification within the NLODP, which resulted in a systematic and uniform collection of data. Together with the systematic sampling procedure of potential sources and the advanced serotyping and genotyping (AFLP and SBT) techniques, this enabled us to further clarify the role of wellness centres in Legionella infections in eight years of Legionnaires' disease source identification efforts in the Netherlands.

Nevertheless, it should be kept in mind that the investigated wellness centres were not a random selection of all available centres in the Netherlands. Sampling of wellness centres was only performed according to the protocol of the NLODP. Furthermore, the ranking of the potential sources of infection that were mentioned by the patients is influenced by the overall presence of particular sources (there are clearly more home residences than wellness centres or car wash installations present in the environment). Random sampling of centres that are not directly linked to Legionnaires' disease patients, for presence of Legionella could further elucidate the contribution of these centres to Legionnaires' disease in the Netherlands. It should also be noted that despite the large number of positive source investigations in wellness centres, only two matches in genotype were found during the eight years of this study period. Although this is partly a reflection of the limited number of clinical isolates that were available for genotype comparison in case of a positive source investigation, a larger number of genotype matches that actually linked cases to wellness centres would have strengthened the evidence for the role of wellness centres in Legionnaires' disease.

In conclusion, wellness centres are not merely the health promoting facilities they are often seen as, but also potential sources for Legionnaires' disease. Despite control measures that are taken after identification of a first patient, some individual centres have been related to an accumulating number of Legionnaires' disease patients over time. This questionable role of wellness centres requires increased attention from wellness centre owners, the VROM Inspectorate, water companies, and Municipal Public Health Services. Furthermore, as many sources remain unknown at the moment this could increase the number of identified sources of Legionnaires' disease.
Financial support

Regional Public Health Laboratory Kennemerland, Haarlem, the Netherlands; National Institute for Public Health and the Environment, Bilthoven, the Netherlands.

\section{References}

1. Blum N, Blum A. Beneficial effects of sauna bathing for heart failure patients. Exp Clin Cardiol. 2007;12(1):29-32.

2. Hannuksela ML, Ellahham S. Benefits and risks of sauna bathing. Am J Med. 2001;110(2):118-26.

3. Den Boer JW, Yzerman E, van Belkum A, Vlaspolder F, van Breukelen FJ. Legionnaires' disease and saunas. Lancet. 1998;351(9096):114.

4. Foster K, Gorton R, Waller J. Outbreak of legionellosis associated with a spa pool, United Kingdom. Euro Surveill. 2006;11(38): pii=3053. Available from: http://eurosurveillance. org/ViewArticle.aspx?Articleld $=3053$

5. Campese C, Roche D, Clément C, Fierobe F, Jarraud, de Waelle $\mathrm{P}$, et al. Cluster of Legionnaires' disease associated with a public whirlpool spa, France, April-May 2010. Euro Surveill 2010;15(26):pii=19602. Available from: http://eurosurveillance. org/ViewArticle. aspx?Articleld $=19602$

6. Muder RR, Yu VL, Fang GD. Community-acquired Legionnaires' disease. Semin Respir Infect. 1989;4(1):32-9.

7. Sopena N, Sabrià M, Pedro-Botet ML, Manterola JM, Matas L, Dominguez J, et al. Prospective study of community-acquired pneumonia of bacterial etiology in adults. Eur J Clin Microbiol Infect Dis. 1999;18(12): 852-8.

8. Braun JJ, de Graaff CS, de Goey J, Zwinderman AH, Petit PL. Buiten het ziekenhuis opgelopen pneumonie: verwekkers en beloop bij patiënten opgenomen in een algemeen ziekenhuis [Community-acquired pneumonia: pathogens and course in patients admitted to a general hospital]. Ned Tijdschr Geneeskd. 2004;148(17):836-40. Dutch.

9. Harrison TG, Afshar B, Doshi N, Fry NK, Lee JV. Distribution of Legionella pneumophila serogroups, monoclonal antibody subgroups and DNA sequence types in recent clinical and environmental isolates from England and Wales (2000-2008). Eur J Clin Microbiol Infect Dis. 2009;28(7):781-91.

10. Fraser DW, Tsai TR, Orenstein W, Parkin WE, Beecham HJ, Sharrar RG, et al. Legionnaires' disease: description of an epidemic of pneumonia. N Engl J Med. 1977;297(22):1189-97.

11. Bhopal RS. Geographical variation of Legionnaires' disease: a critique and guide to future research. Int J Epidemiol. 1993;22(6):1127-36.

12. Den Boer JW, Verhoef L, Bencini MA, Bruin JP, Jansen R, Yzerman EP. Outbreak detection and secondary prevention of Legionnaires' disease: a national approach. Int J Hyg Environ Health. 2007;210(1):1-7.

13. NEN NPR 6569:1992 - Bacteriological examination of water - Explanatory comments on sampling and preservation according to NEN 6559. Delft: The Netherlands Standardization Institute (NEN); 1992.

14. NEN-EN-ISO 19458:2007 - Water quality - sampling for microbiological analysis. Delft: The Netherlands Standardization Institute (NEN); 2007.

15. Joseph C, Lee J, Van Wijngaarden J, Drasar V, Castellani Pastoris M. European Working Group for Legionella Infections. European Guidelines for Control and Prevention of Travel Associated Legionnaires’ Disease. London: Public Health Laboratory Service; Jan 2005. Available from: http://www.hpa. org.uk/web/HPAwebFile/HPAweb_C/1274093149925

16. Fry NK, Bangsborg JM, Bernander S, Etienne J, Forsblom B, Gaia V, et al. Assessment of intercentre reproducibility and epidemiological concordance of Legionella pneumophila serogroup 1 genotyping by amplified fragment length polymorphism analysis. Eur J Clin Microbiol Infect Dis. 2000;19(10):773-80.

17. Gaia V, Fry NK, Afshar B, Lück PC, Meugnier H, Etienne J, et al. Consensus sequence-based scheme for epidemiological typing of clinical and environmental isolates of Legionella pneumophila. J Clin Microbiol. 2005;43(5):2047-52.

18. Ratzow S, Gaia V, Helbig JH, Fry NK, Lück PC. Addition of neuA, the gene encoding $\mathrm{N}$-acylneuraminate cytidylyl transferase, increases the discriminatory ability of the consensus sequence-based scheme for typing Legionella pneumophila serogroup 1 strains. J Clin Microbiol. 2007;45(6):1965-8.

19. Yu VL, Plouffe JF, Pastoris MC, Stout JE, Schousboe M, Widmer $A$, et al. Distribution of Legionella species and serogroups isolated by culture in patients with sporadic community- 
acquired legionellosis: an international collaborative survey. J Infect Dis. 2002;186(1):127-8.

20. Marston BJ, Lipman HB, Breiman RF. Surveillance for Legionnaires' disease. Risk factors for morbidity and mortality. Arch Intern Med. 1994;154(21):2417-22.

21. Joseph CA, Ricketts KD; European Working Group for Legionella Infections. Legionnaires' disease in Europe 2007-2008. Euro Surveill 2010;15(8): pii=19493. Available from: http://www. eurosurveillance.org/ViewArticle.aspx?Articleld=19493

22. Campese C, Bitar D, Jarraud S, Maine C, Forey F, Etienne J, et al. Progress in the surveillance and control of Legionella infection in France, 1998-2008. Int J Infect Dis. 2011;15(1): e30-7. 